\title{
An Effective Multilevel Tabu Search Approach for Balanced Graph Partitioning
}

\author{
Una Benlic and Jin-Kao Hao* \\ LERIA, Université d'Angers \\ 2 Boulevard Lavoisier, 49045 Angers Cedex 01, France \\ To appear in Computers and Operations Research \\ (accepted on October 11, 2010)
}

\begin{abstract}
Graph partitioning is one of the fundamental NP-complete problems which is widely applied in many domains, such as VLSI design, image segmentation, data mining etc. Given a graph $G=(V, E)$, the balanced $k$-partitioning problem consists in partitioning the vertex set $V$ into $k$ disjoint subsets of about the same size, such that the number of cutting edges is minimized. In this paper, we present a multilevel algorithm for balanced partition, which integrates a powerful refinement procedure based on tabu search with periodic perturbations. Experimental evaluations on a wide collection of benchmark graphs show that the proposed approach not only competes very favorably with the two well-known partitioning packages METIS and CHACO, but also improves more than two thirds of the best balanced partitions ever reported in the literature.
\end{abstract}

Keywords: Balanced partitioning; multilevel approach; iterated tabu search.

\section{Introdution}

Given an undirected graph $G=(V, E)$, where $V$ and $E$ denote sets of vertices and edges respectively, the balanced $k$-partitioning problem consists in partitioning the vertex set $V$ into $k(k \geq 2)$ disjoint subsets of approximately equal size, such that the number of cutting edges (i.e. edges whose endpoints belong to different subsets) is minimized. The particular partitioning case when $k$

\footnotetext{
* Corresponding author.

Email addresses: benlic@info.univ-angers.fr (Una Benlic), hao@info.univ-angers.fr (Jin-Kao Hao).
} 
is set to two is often called bisection. The class of partitioning problems is notable for its applicability to a wide range of important applications such as VLSI design [1,26], data mining [30], and image segmentation [24].

The general graph $k$-partitioning problem is NP-complete, and it remains NPcomplete even for the case of bisection $(k=2)$ [11]. Therefore, approximate methods constitute a natural and useful solution approach to address this problem. In recent years, many efforts have been made in devising a number of efficient and powerful heuristics.

One of the most popular graph bisection heuristics is the famous KernighanLin (KL) algorithm [19] that iteratively refines an existing bisection. The idea is to find two subsets of vertices of about the same cardinality, one for each bisection part, and to improve the quality of the bisection by swapping two vertices of the two subsets of the bisection. Fiduccia and Mattheyses [10] presented an improvement of the KL algorithm by introducing the concept of cell gain and an efficient data structure to calculate cell gains. Additional KLlike heuristics are reported, for instance, in [17] for bisection and in [8, 18, 28] for $k$-partitioning. Other well-known algorithms are based on popular metaheuristics such as tabu search [7,23,3], simulated annealing [15], genetic and evolutionary algorithms [27,5,20,25,26].

To handle large and very large graphs, the so-called multilevel paradigm proves to be quite useful $[2,8,18,28,21$. The basic idea of a multilevel approach is to successively create a sequence of progressively smaller graphs by grouping vertices into clusters (coarsening phase). This phase is repeated until the size of the smallest graph falls below a certain coarsening threshold. Afterward, a partition of the coarsest graph is generated (initial partitioning phase), and then successively projected back onto each of the intermediate graphs in reverse order (uncoarsening phase). The partition at each level is improved by a dedicated refinement algorithm before moving to the upper level. Even though almost all multilevel algorithms employ the same or similar heuristics in the coarsening phase, they differ greatly in the way the initial partition is generated, and how the successive partitions are refined throughout each uncoarsening phase.

As illustrated in [29], the multilevel paradigm is a useful approach to solving combinatorial optimization problems. Basically, the approach allows one to approximate the initial problem by solving successively smaller (and easier) problems. Moreover, the coarsening helps filter the solution space by placing restrictions on which solutions the refinement algorithm can visit.

This work is based on the multilevel paradigm. The main contribution reported in this paper is a perturbation-based iterated tabu search procedure which is specially devised for balanced $k$-partitioning and used for partition refinement 
of each coarsened graph. Integrated within the multilevel approach, our Multiplevel Iterated Tabu Search (MITS) algorithm competes very favorably with the two well-known partitioning packages METIS [16] and CHACO [8]. Indeed, extensive experiments performed on the whole set of benchmark graphs from the University of Greenwich graph partitioning archive reveal that MITS is able to find better partitions (with fewer cutting edges) than METIS and CHACO for a large portion of these benchmark graphs. Furthermore, when the running time is prolonged up to one hour, MITS even improves some two thirds of the current best balanced partitions ever reported in the literature while many of these best partitions were obtained by previous algorithms using a significantly longer running time (up to one week).

The rest of the paper is organized as follows. In the next Section, we provide the formal graph partitioning description, as well as some notations used throughout this paper. In Section 3, we describe the multilevel paradigm, and detail the phases of the proposed multilevel algorithm. In Section 4, we present the perturbation-based tabu search algorithm, which is the key partition refinement mechanism in our multilevel approach. In Section 5, we present computation results and comparisons on the benchmark graphs. Finally, in Section 6 we investigate two important features of the the proposed MITS algorithm.

\section{Problem description and notations}

Given an undirected graph $G=(V, E), V$ and $E$ being the set of vertices and edges respectively, and a fixed number $k$, a $k$-partitioning of $G$ can be defined as a mapping (partition function) $\pi: V \rightarrow\{1,2, \ldots, k\}$ that distributes the vertices of $V$ among $k$ disjoint subsets $S_{1} \cup S_{2} \cup \ldots \cup S_{k}=V$ of roughly equal size.

The partition function $\pi$ induces a new graph $G_{\pi}=G_{\pi}\left(S, E_{c}\right)$, where $S=$ $\left\{S_{1}, S_{2} \ldots S_{k}\right\}$ and an edge $\left\{S_{x}, S_{y}\right\} \in E_{c}$ exists if there are two adjacent vertices $u, v \in V$ such that $u \in S_{x}$ and $v \in S_{y}$. The set $E_{c}$ corresponds to the set of cutting edges of $G$ induced by the partition. Vertices $u$ and $v$ of a cutting edge $\{u, v\} \in E_{c}$ are called border vertices of the partition.

Throughout this paper, the initial input graph $G$ is supposed to have a unit cost weight for both vertices and edges. However, as explained in Section 3.2, the multilevel approach generates intermediate (coarsened) graphs where vertices and edges may have different weights (see Section 3.2). As a general rule, these weights increase during the coarsening phase, and decrease during the uncoarsening phase. Let $|v|$ denote the weight of a vertex $v$. Then, the weight $W\left(S_{i}\right)$ of a subset $S_{i}$ is equal to the sum of weights of the vertices in $S_{i}, W\left(S_{i}\right)=\sum_{v \in S_{i}}|v|$. 
The optimization objective $f$ of our balanced partition problem is to find a balanced partition with the minimum sum of weights of cutting edges $E_{c}$.

\section{$3 \quad$ Multilevel Tabu Search graph partitioning}

\subsection{General procedure}

Our Multilevel Tabu Search approach follows the general mulitlevel paradigm [8, 42 .

Let $G_{0}=\left(V_{0}, E_{0}\right)$ be the initial graph, and let $k$ denote the number of partition subsets. The multilevel paradigm can be summarized by the following steps.

(1) Coarsening phase: The initial graph $G_{0}$ is transformed into a sequence of smaller graphs $G_{1}, G_{2}, \ldots, G_{m}$ such that $\left|V_{0}\right|>\left|V_{1}\right|>\left|V_{2}\right|>\ldots>$ $\left|V_{m}\right|$. This phase stops when $\left|V_{m}\right|$ reaches a fixed threshold (coarsening threshold).

(2) Partitioning phase: A $k$-partition $P_{m}$ of the coarsest graph $G_{m}=\left(V_{m}, E_{m}\right)$ is generated.

(3) Uncoarsening phase: Partition $P_{m}$ is progressively projected back to each intermediate $G_{i}(i=m-1, m-2, \ldots, 0)$. Before each projection, the partition is first refined (improved) by a refinement algorithm.

This process leads thus to a sequence of partitions $P_{m}, P_{m-1}, P_{m-2}, \ldots P_{0}$. The last one, i.e. $P_{0}$ is returned as the final partition of the original graph $G_{0}$.

Our Multilevel Iterated Tabu Search (MITS) algorithm follows this general scheme and is described in Algorithm 1.

\subsection{Coarsening phase}

Creating a coarser graph $G_{i+1}=\left(V_{i+1}, E_{i+1}\right)$ from $G=\left(V_{i}, E_{i}\right)$ consists in finding an independent subset of graph edges $\Gamma \subset E_{i}$, and then collapsing the two vertices of each edge of $\Gamma$. A set of edges is independent if no two edges in the set are incident to the same vertex, which implies that exactly two vertices are collapsed each time. If a vertex is not incident to any edge of subset $\Gamma$, it is simply copied over to $G_{i+1}$.

When vertices $v_{1}, v_{2} \in V_{i}$ are collapsed to form a vertex $v_{c} \in V_{i+1}$, the weight of the resulting vertex $v_{c}$ is set equal to the sum of weights of vertices $v_{1}$ and $v_{2}$. The edge that is incident to $v_{c}$ becomes the union of all the edges 


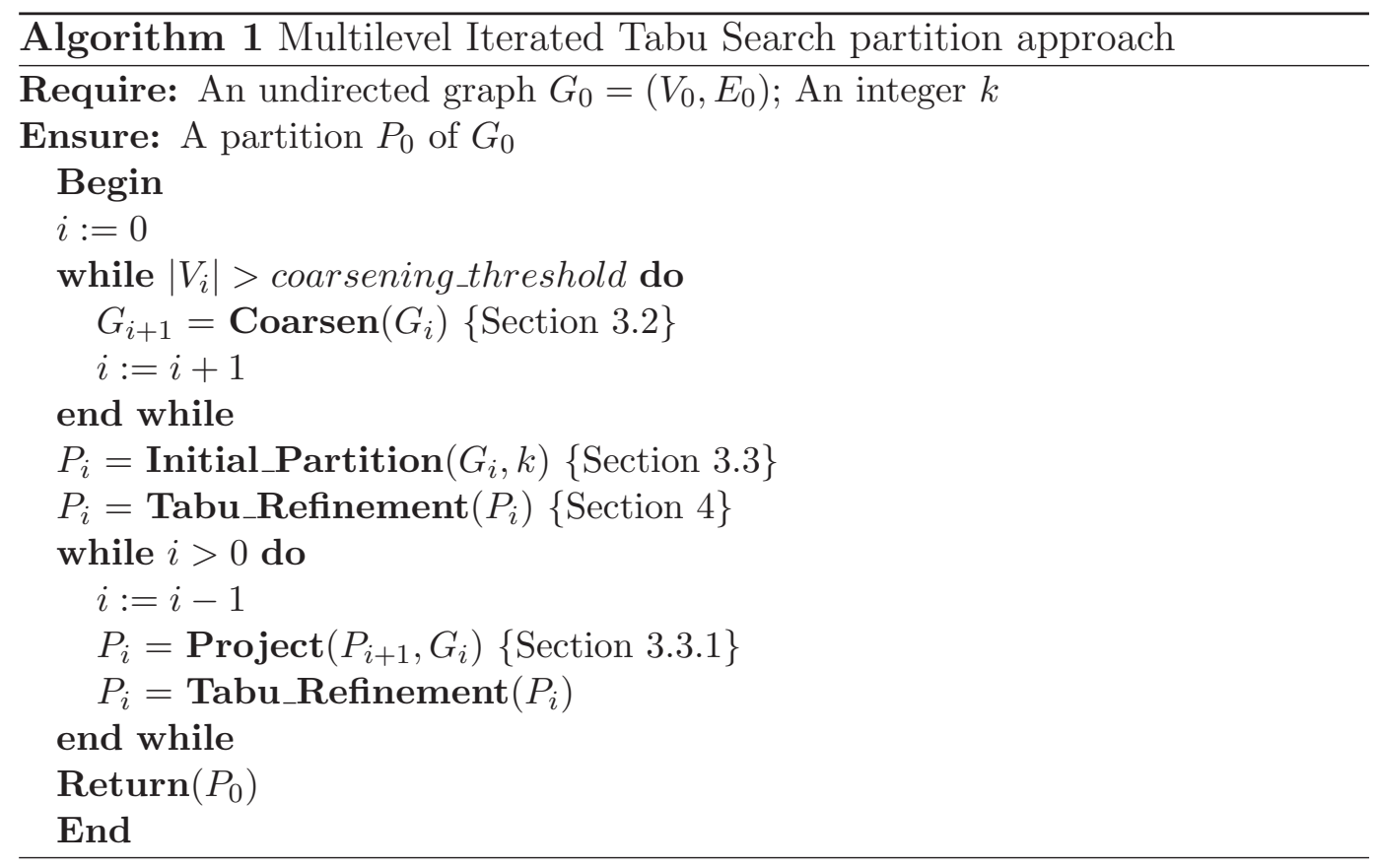

incident to $v_{1}$ and $v_{2}$, minus the edge $\left\{v_{1}, v_{2}\right\} \in E_{i}$. Therefore, during the coarsening process, both the vertex and edge weight increases. At any level of the coarsening phase, the weight of a vertex (an edge) corresponds to the number of aggregated vertices (edges) of the initial graph $G_{0}$.

One key issue here is the selection of the independent subset of graph edges $\Gamma$ to be collapsed at each step of the coarsening phase. This can be achieved by finding a maximum matching of the graph. Recall that a matching of a graph is a subset of edges such that no two edges share the same vertex [22]. There exist polynomial time algorithms for tackling this problem, with running time of at least $O\left(|V|^{2.5}\right)$. Unfortunately, this is too slow to be applicable to the partitioning problem. That is why we compute maximal matching using an edge contraction heuristic called heavy-edge matching (HEM), which has $O(|E|)$ time complexity [17]. This method considers vertices in random order, matching each unmatched vertex $v$ with its unmatched neighbor $u$, if any, such that the weight of edge $\{u, v\}$ is maximum among all the edges incident to $v$.

\subsection{Initial partition and its refinement}

Once we have obtained a sequence of smaller graphs in the coarsening phase, the next step is to generate an initial partition of the smallest graph $G_{m}=$ $\left(V_{m}, E_{m}\right)$. The initial partitioning phase of our algorithm consists of first assigning randomly the vertices of $V_{m}$ to $k$ subsets $S_{i} \in\left\{S_{1}, S_{2} \ldots S_{k}\right\}$, such that the number of clustered vertices in each subset is evenly balanced, i.e. the number of represented vertices $n$ of $G_{0}$ in each subset $\left\{S_{1}, S_{2} \ldots S_{k}\right\}$ is $n \leq\left\lceil\left|V_{m}\right| / k\right\rceil$. 
Afterward, we refine this initial partition by means of a dedicated PerturbationBased Tabu Search algorithm which is described in Section 4. This refinement step is essential for our approach to improve progressively the quality of partitions. It should be noted that for certain graphs, it is impossible to obtain a perfectly balanced initial partition, since weights of vertices in the coarsest graph can be greatly inhomogeneous. The imbalance $\varepsilon$ of a partition $\left\{S_{1}, S_{2} \ldots S_{k}\right\}$ is defined as $\varepsilon=\max _{i \in\{1 . . k\}} W\left(S_{i}\right) / W_{\text {opt }}$ where $W_{\text {opt }}=\lceil|V| / k\rceil$, $\lceil x\rceil$ being the ceiling function returning the first integer $\geq x$.

This imbalance is gradually reduced throughout each uncoarsening step, and (usually) completely eliminated by the end of the algorithm execution.

\subsubsection{Uncoarsening phase}

The uncoarsening phase carries out the inverse of the coarsening phase. The idea is to go from level to level, uncoarsening the clustered vertices in the opposite way they were grouped during the coarsening phase. The partition projection from a graph $G_{i}=\left(V_{i}, E_{i}\right)$ onto a partition of the parent graph $G_{i-1}=\left(V_{i-1}, E_{i-1}\right)$ is a trivial process. If a vertex $v \in V_{i}$ is in subset $S_{m}$ of the partition of $G_{i}$, then the matched pair of vertices $v_{1}, v_{2} \in V_{i-1}$ which represents vertex $v \in V_{i}$ will be in subset $S_{m}$ of the projected partition of $G_{i-1}$.

Before projecting a partition to the next level, we apply the perturbationbased tabu search refinement algorithm (Section 4) to improve the partition quality. As the uncoarsening/refining process proceeds, the partition quality of graph $G_{i-1}$ is usually better than that of $G_{i}$ because there is a greater degree of freedom for refinement. This is one of the most attractive characteristics of a multilevel algorithm.

\section{Partition refinement by Perturbation-based Iterated Tabu Search}

The refinement algorithm, used at each level for partition improvement, is based on a perturbation-based iterated tabu search procedure. Basically, the tabu search algorithm [13] ensures the intensification of the approach, while the perturbation mechanism brings controlled diversification into the search. Experiments show that the combination of these two mechanisms constitutes a very effective refinement method for obtaining high quality partitions. Algorithm 2 describes this refinement procedure, whose components are detailed in the following sections. 
Algorithm 2 Perturbation-based Tabu Search for Partition Refinement

Require: Graph $G_{i}=\left(V_{i}, E_{i}\right)$ at level $i$; Initial partition $P_{i}=\left\{S_{1}, S_{2} \ldots S_{k}\right\}$.

Ensure: The best partition $P_{i} *$ of graph $G_{i}$

1: Initiate iteration_counter, tabu_list, tabu_tenure, move_frequency_list

2: $P_{i}^{*} \leftarrow P_{i}\left\{P_{i}^{*}\right.$ records the best partition found so far $\}$

3: for each vertex $v$ and each subset $S_{m}$ of $P_{i}$ such that $v \notin S_{m}$ do

4: $\quad$ Compute move gain $g(v, m)$

5: $\quad$ Insert $v$ into the bucket structure corresponding to $S_{m}$ \{Section 4.1.3 $\}$

6: end for

7: repeat

8: $\quad$ Apply neighborhoods $N_{1}$ and $N_{2}$ in token-ring way (Section 4.1.2) $\}$

9: $\quad$ if turn to employ $N_{1}$ then

10: $\quad$ Apply move operator Single_Move $\}$ SSections 4.1.2 and 4.1.4\}

11: $\quad$ Select target subset $S_{m}$ and vertex $v_{m}$ for migration

12: $\quad P_{i}:=P_{i} \oplus \operatorname{Move}\left(v_{m}, S_{m}\right)\left\{\right.$ Move $v_{m}$ to $S_{m}$ to generate new partition

13: $\quad$ Update tabu list and bucket sorting structure

14: $\quad$ else if turn to empoy $N_{2}$ then

15: $\quad$ Apply move operator Double_Move\} \{Sections 4.1.2 and 4.1.4 \}

16: $\quad$ Select target subset $S_{m}$ and vertex $v_{m}$ for migration

17: $\quad P_{i}:=P_{i} \oplus \operatorname{Move}\left(v_{m}, S_{m}\right)$

18: $\quad$ Select target subset $S_{n}$ and vertex $v_{n}$ for migration

19: $\quad P_{i}:=P_{i} \oplus \operatorname{Move}\left(v_{n}, S_{n}\right)$

20: $\quad$ Update tabu list and bucket sorting structure

21: end if

22: $\quad$ U Update the best solution $P_{i}^{*}$ found so far

23: $\quad$ if $\left(f\left(P_{i}\right) \leq f\left(P_{i}^{*}\right)\right)$ and $P_{i}$ is at least as well balanced as $P_{i}^{*}$ then

24: $\quad P_{i}^{*} \leftarrow P_{i}$

25: $\quad$ end if

26: $\quad$ if $\left(P_{i}^{*}\right.$ not improved after $\gamma$ iterations) then

27: $\quad P_{i}:=\operatorname{Perturb}\left(P_{i}, p_{\text {str }}\right)\{$ Section 4.2 $\}$

28: $\quad$ end if

29: until stop condition not met

\subsection{Tabu Search}

In this subsection, we focus on the tabu search engine of our partition refinement algorithm. It explores the search space by repeatedly replacing the current solution with a best non-recently visited neighboring solution, even if the later deteriorates the solution quality. Since tabu search relies on the belief that intelligent search should be based on learning, its underlying element is the usage of flexible memory that exploits and keeps track of the search history. In order to avoid possible cycling and go beyond local optimum, tabu search introduces the notion of tabu list to forbid the recently visited solutions. 


\subsubsection{Objective and partition balance}

The optimization objective $f$ of our $k$-partitioning problem is to minimize the sum of weights of cutting edges of a balanced partition. Note that, in the given problem formulation, partition balance is imposed as a constraint. However, as we have already mentioned earlier, it is often impossible to establish perfect balance in coarsened graphs, since vertex weights can be extremely inhomogeneous. It is during the partition refinement of the levels which are closer to the original graph that the balance condition is (usually) completely satisfied.

More precisely, our refinement algorithm uses two move operators that not only help optimize the objective (number of cutting edges), but also take care of partition imbalance (see Section 4.1.2). Typically, these move operators transfer vertices to subsets with smaller weight. Applying these directional moves generally allows the search to progressively establish perfect partition balance towards the end of the backward projection process.

During the refinement process, the current solution $P_{i}$ ( $k$-partition) becomes the new best solution $P^{*}$ only if $P_{i}$ is at least as good as $P^{*}$ in terms of the optimization objective and partition balance (see lines 23-25 of Algorithm 1).

\subsubsection{Neighborhoods and their exploration}

Our tabu search algorithm employs two neighborhood relations $N_{1}$ and $N_{2}$ that are based on two different move operators. These operators transfer respectively one and two vertices between subsets of a partition.

To define these move operators, we first introduce the notion of move gain which indicates how much a partition is improved according to the optimization objective if a vertex is moved to another subset. Given a vertex $v$ from subset $S_{m}$, the gain $g(v, n)$ can be computed for every other subset, $S_{n}, m \neq n$. As we show in 4.1.3, the vertex with the best (highest) gain can be easily determined using a special bucket data structure.

Let $P=\left\{S_{1}, S_{2} \ldots S_{k}\right\}$ be a $k$-partition, and let $S_{\max }$ be the subset with the

maximum weight, $S_{\max }=\max _{i \in\{1 . . k\}}\left\{W\left(S_{i}\right)\right\}$. The two move operators are given below.

Single_Move $\left(N_{1}\right)$ : Move one highest gain vertex $v_{m}$. Choose randomly a subset $S_{m}, m \neq \max$. Then, select a highest gain vertex $v_{m}$ (see Section 4.1.4) whose current subset is $S_{c}$, such that $S_{c} \in\left\{S_{i} \in P \mid W\left(S_{i}\right)>W\left(S_{m}\right)\right\}$. Finally, move the selected vertex $v_{m}$ to the target subset $S_{m}$.

Double_Move $\left(N_{2}\right)$ : Move two highest gain vertices $v_{m}$ and $v_{n}$. Choose vertex $v_{m}$ and its target subset $S_{m}$ as for Single_Move. Choose randomly 
another target subset $S_{n} \in P, n \neq \max$ and $n \neq m$. Then, select a highest gain vertex $v_{n}$ whose current subset is $S_{c}$, such that $S_{c} \in\left\{S_{i} \in P \mid S_{i} \neq\right.$ $\left.S_{n}, S_{i} \neq S_{m}\right\}$. Move $v_{m}$ to $S_{m}$, and $v_{n}$ to $S_{n}$.

It is important to mention that a vertex $v$ is considered to be moved to subset $S_{i}$ if and only if $v$ is adjacent to at least one vertex of $S_{i}(v$ is a border vertex relative to $S_{i}$ ). Otherwise, moving $v$ to $S_{i}$ does not make any sense and would only deteriorate the partition quality. Our move operators focus thus on these critical vertices, reducing considerably the number of candidate moves to be examined at each iteration of the search process. Consequently, given the bucket data structure from Section 4.1.3, the worst-case complexity of choosing a vertex $v$ to be moved to a subset $S_{i}$ with any of the two move operators is equal to the number of border vertices relative to $S_{i}$.

Also note that these move operators progressively lead the search toward a balanced partition since basically they constraint (partially with Double_Move) vertex migration from heavy weight subsets to light weight subsets. Indeed, with Single_Move and the first choice of Double_Move, a vertex can never move to a subset with a higher weight. The second choice of Double_Move is allowed to bring some diversification into the search.

In the case of $k$-partitioning $(k>2)$, the two neighborhoods $N_{1}$ and $N_{2}$ are jointly explored by the tabu search algorithm in a token-ring way. In tokenring search [12], one neighborhood search is applied to the local optimum produced by the previous one, and this process continues until no improvement is possible. In the case of bisection $(k=2)$, the neighborhood $N_{2}$ is not suitable and only $N_{1}$ is employed.

\subsubsection{Bucket sorting}

To ensure a fast evaluation of the neighborhoods, our implementation uses a bucket data structure which keeps vertices ordered by their gains. This data structure is used to avoid unnecessary search for the highest gain vertex and to minimize the time needed for updating the gains of vertices affected by each move.

The bucket sorting structure was first proposed by Fiduccia and Mattheyses [10] to improve the Kerninghan-Lin algorithm [19] for graph bisection. It consists in placing all vertices with the same gain $g$ in a bucket that is ranked $g$. Then, finding a vertex with the maximum gain simply consists in finding the non-empty bucket with the highest rank, and selecting a vertex from the bucket (in MITS a vertex is selected from this bucket according to a policy detailed in Section 4.1.4). After each move, the bucket structure is updated by recomputing gains of the selected vertex and its neighbors, and transferring these vertices to appropriate buckets. The bucket data structure, as suggested 
in [10, maintains two arrays of buckets, one for each subset of a bisection, and is not suitable for $k$-partitioning.

We propose an adaptation of this idea for $k$-partitioning, designed to be used with the two neighborhood relations $N_{1}$ and $N_{2}$. In our implementation, we maintain $k$ arrays of buckets, one for each subset of the $k$-partition, where each bucket of an array $i, 1 \leq i \leq k$ is (as usually) represented by a double linked list. Each doubly linked list contains vertices with the gain corresponding to the rank of the link list in the given array of buckets. As proposed in [10], we also keep an additional array of vertices where each element (vertex) points to its corresponding vertex in the doubly linked lists. This enables a direct access to the vertices in doubly linked lists of each array. During the update of gains, this data structure also enables the transfer of vertices from one bucket to another in constant time.

In the search process, only border vertices are considered, and thus included in the bucket data structure. Therefore, given a vertex $v$ with its current subset $S_{c}$, for every other subset $S_{m}, m \neq c$, we calculate the gain $g(v, m)$ in constant time, and place vertex $v$ to the array of buckets corresponding to subset $S_{m}$ only if there is at least one vertex in $S_{m}$ adjacent to $v$, i.e. $v$ is a border vertex relative to $S_{m}$. The gains of all the vertices adjacent to $v$ are recalculated (in $O(1)$ ) and updated in the bucket structure in constant time (delete and insert operations in the bucket are both of $O(1)$ complexity). Therefore, the complexity of moving vertex $v$ from $S_{c}$ to a target subset $S_{m}$ is equal to the number of vertices adjacent to $v$.

According to the complexity analysis, $k$ has no influence on the performance of the proposed algorithm in terms of computing time. However, it does require a greater amount of memory as $k$ increases.

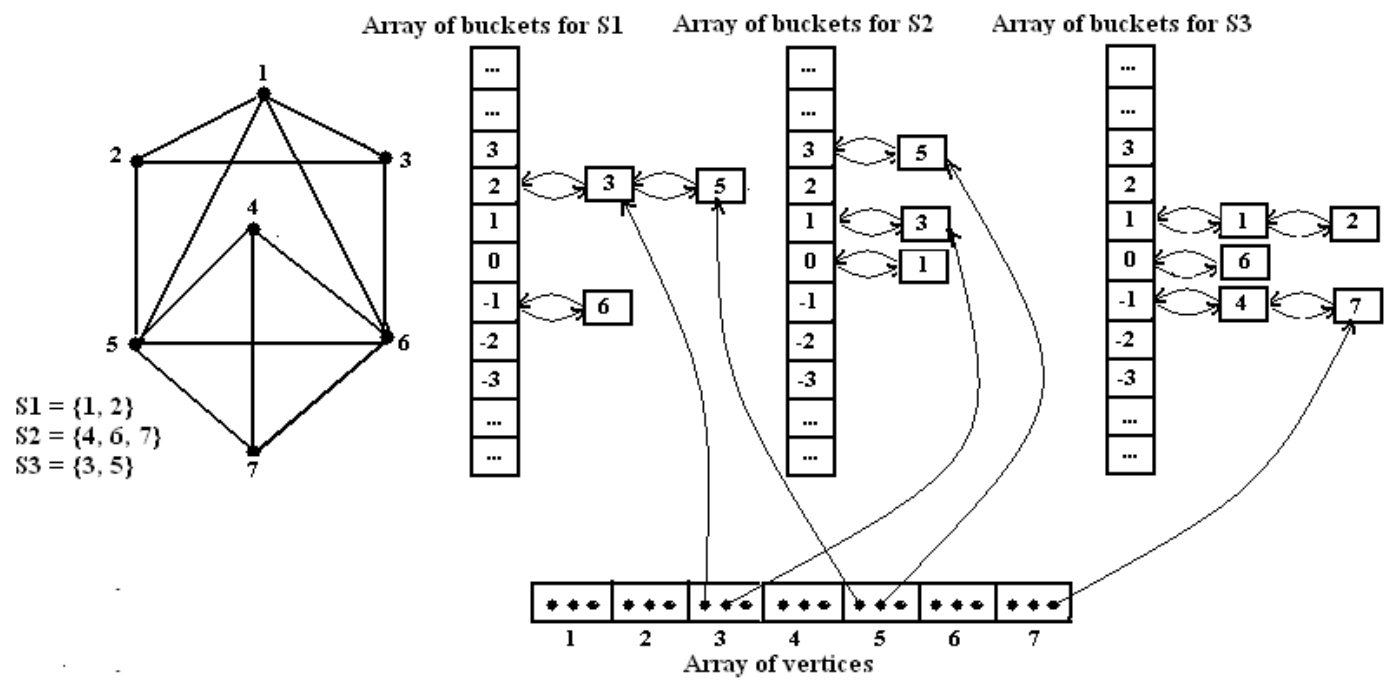


Figure 1: An example of the bucket structure for 3-partitioning

An illustration of the proposed bucket sort data structure for 3-partitioning of a graph with 7 vertices is provided in Figure 1 .

\subsubsection{Selection strategy for vertex migration}

As previously explained, when the non-empty bucket with the highest rank contains more than one vertex, a selection strategy is needed to determine the vertex $v_{m}$ (and $v_{n}$ ) to be transferred to $S_{m}$ (and $v_{n}$ to $S_{n}$ ). Our selection strategy integrates several pieces of history information in order to make this choice as pertinent as possible.

The selection strategy is first conditioned by the tabu status (see 4.1.5). Let $V_{\text {cand }}$ be the subset of candidate vertices (with the highest gain) for migration to subset $S_{m}$. A vertex $v \in V_{\text {cand }}$, whose current subset is $S_{c}$, is considered for migration to $S_{m}$ if it is not tabu or moving $v$ to $S_{m}$ leads to a new partition better than the best partition $P^{*}$ found so far (this later case is called aspiration in the tabu search terminology).

The vertex selection strategy employs two additional criteria which are based on vertex move frequency and vertex weight. The move frequency is a long term memory that records, for each vertex $v$, the number of times $v$ has been moved to a different subset. Our usage of this frequency information penalizes moves with vertices having high frequency count, by giving priority to those that have been moved less often. This long term memory is reset to zero only before the beginning of each refinement phase (see line 2 in Algorithm 1). This strategy favors the diversification of the search.

If there is more than one vertex with the same move frequency in the set $V_{\text {cand }}$, we use the second criterion to distinguish them and prefer a vertex $v$ which, when moved to subset $S_{m}$, minimizes the weight difference between the target subset $S_{m}$ and the original subset $S_{c}$. This strategy helps to improve the partition balance.

\subsubsection{Tabu list and tabu tenure management}

Each time a vertex $v$ is moved from a subset $S_{c}$ to another subset $S_{m}$, it is forbidden to move $v$ back to its original subset $S_{c}$ for the next $t t$ iterations (tabu tenure). The tabu tenure $t t$ of the move $\left(v, S_{c}\right)$ is tuned dynamically according to the number of border vertices relative to $S_{c}$,

$$
t t\left(v, S_{c}\right)=\left|V\left(S_{c}\right)\right| * \alpha+\operatorname{random}(0,2),
$$


where $\left|V\left(S_{c}\right)\right|$ is the number of border vertices relative to $S_{c}, \alpha$ a coefficient from $\{0.05,0.1,0.2,0.3\}$, and $\operatorname{random}(0,2)$ a random integer number in the range $[0,2]$.

\subsection{Perturbation mechanism}

The tabu search algorithm described above employs an aggressive search strategy to explore the search space. Indeed, for a transition from the current partition to a new partition, only border vertices relative to the target subset $S_{m}$ are considered. Such a consideration helps the search to focus on critical vertices and improve rapidly the solution quality.

To complement this search strategy, a perturbation mechanism is applied to bring diversification into the search, if the best partition $P^{*}$ is not improved during $\gamma$ iterations (see lines 26-28 of Algorithm 2). The perturbation consists in moving $p_{\text {str }}$ selected vertices (perturbation strength) to a given subset.

Precisely, let $\left\{S_{1}, S_{2} \ldots S_{k}\right\}$ be the current partition and let $S_{\max }$ be the subset with the maximum vertex weight, $S_{\max }=\max _{i \in\{1 \ldots k\}}\left\{W\left(S_{i}\right)\right\}$. Then the perturbation phase is defined as follows.

(1) Randomly select a subset $S_{m} \in\left\{S_{1}, S_{2}, \ldots, S_{k}\right\}-\left\{S_{\max }\right\}$.

(2) Randomly choose a vertex $v$ whose current subset is $S_{c}$, such that $S_{c} \in$ $\left\{S \mid W(S)>W\left(S_{m}\right)\right\}$.

(3) Move the selected vertex $v$ to subset $S_{m}$.

(4) Repeat steps (1) to (3) $p_{\text {str }}$ times.

The described mechanism is similar to the neighborhood relation $N_{1}$. However, there is a significant difference. Not only does it neglect the tabu status of a vertex, but it also allows a vertex $v$ to be moved to subset $S_{m}$ even if it is not a border vertex relative to $S_{m}$. This leads the search to new areas and helps to escape from deep local optima (see also Section 6).

\section{$5 \quad$ Experimental results}

\subsection{Benchmark instances}

To evaluate the efficiency of the proposed approach, we carry out extensive experiments on a set of well-known benchmark graphs that are frequently used to assess graph partitioning algorithms. These graphs correspond to real-life problems arising in different applications. They can be downloaded from the 
Table 1

The list of benchmark graphs together with their characteristics

\begin{tabular}{|c|c|c|c|c|c|c|}
\hline Graph & $\begin{array}{l}\text { Size } \\
\qquad|V|\end{array}$ & $|E|$ & $\begin{array}{l}\text { Degre } \\
\text { Max }\end{array}$ & Min & Avg & Type \\
\hline add 20 & 2395 & 7462 & 123 & 1 & 6.23 & 20-bit adder \\
\hline data & 2851 & 15093 & 17 & 3 & 10.59 & 3D FEM \\
\hline 3elt & 4720 & 13722 & 9 & 3 & 5.81 & 2D nodal graph \\
\hline uk & 4824 & 6837 & 3 & 1 & 2.83 & 2D dual graph \\
\hline add32 & 4960 & 9462 & 31 & 1 & 3.82 & 32-bit adder \\
\hline bcsstk33 & 8738 & 291583 & 140 & 19 & 66.74 & 3D stiffness matrix \\
\hline whitaker3 & 9800 & 28989 & 8 & 3 & 5.92 & 2D nodal graph \\
\hline crack & 10240 & 30380 & 9 & 3 & 5.93 & 2D nodal graph \\
\hline wing_nodal & 10937 & 75488 & 28 & 5 & 13.80 & 3D nodal graph \\
\hline fe_4elt2 & 11143 & 32818 & 12 & 3 & 5.89 & 2D FEM \\
\hline vibrobox & 12328 & 165250 & 120 & 8 & 26.8 & Sparse matrix \\
\hline bcsstk29 & 13992 & 302748 & 70 & 4 & 43.27 & $3 \mathrm{D}$ stiffness matrix \\
\hline 4 elt & 15606 & 45878 & 10 & 3 & 5.88 & 2D nodal graph \\
\hline fe_sphere & 16386 & 49152 & 6 & 4 & 5.99 & 3D FEM \\
\hline cti & 16840 & 48232 & 6 & 3 & 5.73 & 3D semi-structured graph \\
\hline memplus & 17758 & 54196 & 573 & 1 & 6.10 & Memory circuit \\
\hline $\operatorname{cs} 4$ & 22499 & 43858 & 4 & 2 & 3.90 & 3D nodal graph \\
\hline bcsstk30 & 28924 & 1007284 & 218 & 3 & 69.65 & $3 \mathrm{D}$ stiffness matrix \\
\hline bcsstk31 & 35588 & 572914 & 188 & 1 & 32.197 & $3 \mathrm{D}$ stiffness matrix \\
\hline fe_pwt & 36519 & 144794 & 15 & 0 & 7.93 & 3D FEL \\
\hline bcsstk32 & 44609 & 985046 & 215 & 1 & 44.1636 & $3 \mathrm{D}$ stiffness matrix \\
\hline fe_body & 45097 & 163734 & 28 & 0 & 7.26 & 3D FEM \\
\hline $\mathrm{t} 60 \mathrm{k}$ & 60005 & 89440 & 3 & 2 & 2.98 & 2D dual graph \\
\hline wing & 62032 & 121544 & 4 & 2 & 2.57 & 3D dual graph \\
\hline brack2 & 62631 & 366559 & 32 & 3 & 11.71 & 3D nodal graph \\
\hline finan512 & 74752 & 261120 & 54 & 2 & 6.99 & stochastic programming matrix \\
\hline fe_tooth & 78136 & 452591 & 39 & 3 & 11.58 & 3D FEM \\
\hline fe_rotor & 99617 & 662431 & 125 & 5 & 13.30 & 3D FEM \\
\hline $598 \mathrm{a}$ & 110971 & 741934 & 26 & 5 & 13.37 & 3D FEM \\
\hline fe_ocean & 143437 & 409593 & 6 & 1 & 5.71 & 3D dual graph \\
\hline
\end{tabular}

University of Greenwich Graph Partitioning Archive 11 in the same format as used by JOSTLE, CHACO and METIS. Table 1 provides the whole set of the graphs together with their sizes, vertex degrees and types.

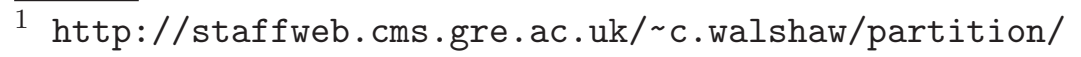


Table 2

Settings of important parameters

\begin{tabular}{ccc}
\hline Parameters & Description & Values \\
\hline$c t$ & coarsening threshold & 200 \\
$\alpha$ & tabu tenure management factor & {$[0.05,0.1,0.2,0.3]$} \\
$p_{s t r}$ & perturbation strength & $0.02 *|V|$ \\
$\gamma$ & non-improvement TS iterations before perturbation & $0.01 *|V|$ \\
\hline
\end{tabular}

\subsection{Experimental protocol}

Our partition algorithm is programmed in $\mathrm{C}++$, and compiled with GNU gcc on a Xeon E5440 with $2.83 \mathrm{GHz}$ and 8GB.

To assess the performance of our MITS algorithm, we report computational results of two experiments: a comparison with two state-of-art graph partitioning packages (METIS [16] and CHACO [8]), and a comparison with the best partitions ever reported in the literature. The parameter settings of MITS applied in both experiments are given in Table 2, and are determined by a preliminary experiment using a small number of graph instances.

\subsection{Comparison with the state-of-art solvers}

In this section, we compare the partitions obtained by our approach in short computing time (limited to 2.5 minutes), with those obtained with the two packages METIS and CHACO. In both cases, we use the latest versions (METIS-4.0, CHACO-2.2). For METIS, we use the multilevel pMetis algorithm, and for CHACO we choose the multilevel KL algorithm with recursive bisection and a coarsening threshold of 100. Since pMetis and CHACO do not allow repeating runs in a randomized way, we run our MITS algorithm only once. In addition, we fix the parameter $\alpha$ (see Section 4.1.5) to 0.1. The cutoff limit depends on the size of graph, and is from 1 seconds (for graphs with up to 3000 vertices) to 2.5 minutes (for the largest graph $f e_{-}$ocean).

Tables 3 and 4 present, for each graph and each value of $k$, the number of cutting edges of the partition obtained with the packages and our proposed algorithm. METIS, CHACO and our approach are labeled as pMetis, CHACO and MITS respectively. The last row in each table with heading Total gives the number of times each algorithm produced the best partition over the 30 benchmark graphs. From tables 3 and 4, we observe that for each value of $k$ MITS performes far better than either of the two packages in terms of partition quality.

From these tables, we also note that for $k \geq 32$, some partitions found with 
Table 3

Comparison of our approach with pMetis and CHACO for $k$ equal to 2,4 and 8

\begin{tabular}{|c|c|c|c|c|c|c|c|c|c|}
\hline \multirow[b]{2}{*}{ Graph } & \multicolumn{3}{|l|}{$\mathrm{k}=2$} & \multicolumn{3}{|l|}{$\mathrm{k}=4$} & \multicolumn{3}{|l|}{$\mathrm{k}=8$} \\
\hline & pMetis & CHACO & MITS & pMetis & $\mathrm{CHACO}$ & METIS & pMetis & CHACO & MITS \\
\hline add 20 & 729 & 742 & 708 & 1292 & 1329 & 1224 & 1907 & 1867 & 1750 \\
\hline data & 218 & 199 & 189 & 480 & 433 & 427 & 842 & 783 & 679 \\
\hline 3elt & 108 & 103 & 90 & 231 & 234 & 214 & 388 & 389 & 352 \\
\hline $\mathrm{uk}$ & 23 & 36 & 23 & 67 & 69 & 47 & 101 & 119 & 113 \\
\hline add32 & 21 & 11 & 11 & 42 & 56 & 40 & 81 & 115 & 74 \\
\hline bcsstk33 & 10205 & 10172 & 10171 & 23131 & 23723 & 22492 & 40070 & 39070 & 34568 \\
\hline whitaker3 & 135 & 131 & 127 & 406 & 425 & 385 & 719 & 765 & 672 \\
\hline crack & 187 & 225 & 184 & 382 & 445 & 371 & 773 & 777 & 720 \\
\hline wing_nodal & 1820 & 1823 & 1797 & 4000 & 4022 & 3715 & 6070 & 6147 & 5481 \\
\hline fe_4elt2 & 130 & 144 & 130 & 359 & 402 & 423 & 654 & 718 & 621 \\
\hline vibrobox & 12427 & 11367 & 11184 & 21471 & 21774 & 19811 & 28177 & 33362 & 24840 \\
\hline bcsstk 29 & 2843 & 3140 & 2852 & 8826 & 9202 & 8572 & 16555 & 18158 & 17014 \\
\hline 4 elt & 154 & 158 & 139 & 406 & 433 & 390 & 635 & 688 & 615 \\
\hline fe_sphere & 440 & 424 & 386 & 872 & 852 & 774 & 1330 & 1302 & 1243 \\
\hline cti & 334 & 372 & 366 & 1113 & 1117 & 1039 & 2110 & 2102 & 1838 \\
\hline memplus & 6337 & 7549 & 5696 & 10559 & 11535 & 9982 & 13110 & 14265 & 12642 \\
\hline $\operatorname{cs} 4$ & 414 & 517 & 377 & 1154 & 1166 & 987 & 1746 & 1844 & 1529 \\
\hline bcsstk30 & 6458 & 6563 & 9812 & 17685 & 17106 & 22436 & 36357 & 37406 & 36373 \\
\hline bcsstk31 & 3638 & 3391 & 2820 & 8770 & 9199 & 8751 & 16012 & 15551 & 15262 \\
\hline fe_pwt & 366 & 362 & 360 & 738 & 911 & 1249 & 1620 & 1670 & 1531 \\
\hline bcsstk32 & 5672 & 6137 & 6936 & 12205 & 15704 & 9864 & 23601 & 25719 & 24435 \\
\hline fe_body & 311 & 1036 & 271 & 957 & 1415 & 728 & 1348 & 2277 & 1293 \\
\hline $\mathrm{t} 60 \mathrm{k}$ & 100 & 91 & 86 & 255 & 235 & 226 & 561 & 524 & 522 \\
\hline wing & 950 & 901 & 861 & 2086 & 1982 & 1770 & 3205 & 3174 & 2686 \\
\hline brack2 & 738 & 976 & 731 & 3250 & 3462 & 3291 & 7844 & 8026 & 7644 \\
\hline finan512 & 162 & 162 & 162 & 324 & 325 & 405 & 810 & 648 & 729 \\
\hline fe_tooth & 4297 & 4642 & 3827 & 8577 & 8430 & 7460 & 13653 & 13484 & 12083 \\
\hline fe_rotor & 2190 & 2151 & 2122 & 8564 & 8215 & 7765 & 15712 & 15244 & 13558 \\
\hline $598 \mathrm{a}$ & 2504 & 2465 & 2402 & 8533 & 8975 & 8159 & 17276 & 17530 & 16270 \\
\hline fe_ocean & 505 & 499 & 468 & 2039 & 2110 & 2850 & 4516 & 5309 & 4272 \\
\hline Total & 7 & 2 & 26 & 5 & 1 & 24 & 4 & 1 & 25 \\
\hline
\end{tabular}

pMetis and our approach are not perfectly balanced. For these cases, we indicate the partition imbalance in parentheses. As it will be seen from the second experiment, MITS in some cases requires more computation time to establish partition balance. Unlike pMetis and our algorithm, CHACO generates perfectly balanced partitions for every value of $k$ since it uses recursive bisection. 
Table 4

Comparison of our approach with pMetis and CHACO for $k$ equal to 16, 32 and 64

\begin{tabular}{|c|c|c|c|c|c|c|c|c|c|}
\hline & $\mathrm{k}=16$ & & & $\mathrm{k}=32$ & & & $\mathrm{k}=64$ & & \\
\hline Graph & pMetis & $\mathrm{CHACO}$ & MITS & pMetis & $\mathrm{CHACO}$ & MITS & pMetis & $\mathrm{CHACO}$ & MITS \\
\hline $\operatorname{add} 20$ & 2504 & 2297 & 2120 & NAN & 2684 & $2524(1.03)$ & $3433(1.07)$ & 3349 & $3219(1.03)$ \\
\hline data & 1370 & 1360 & 1167 & $2060(1.01)$ & 2143 & $1933(1.01)$ & 3116 (1.03) & 3145 & $2924(1.07)$ \\
\hline 3elt & 665 & 660 & 585 & 1093 & 1106 & 1053 & 1710 & 1722 & 1606 \\
\hline $\mathrm{uk}$ & 189 & 211 & 163 & 316 (1.01) & 343 & $296(1.01)$ & $495(1.02)$ & 540 & 496 (1.03) \\
\hline add32 & 128 & 174 & 143 & $288(1.01)$ & 303 & 266 & $626(1.02)$ & 730 & $\mathbf{5 7 1}(1.01)$ \\
\hline bcsstk33 & 59791 & 61890 & 55538 & 86008 & 84613 & 90438 & 116203 & 115530 & 131639 \\
\hline whitaker3 & 1237 & 1218 & 1120 & 1891 & 1895 & 1758 & $2796(1.01)$ & 2811 & 2628 \\
\hline crack & 1255 & 1253 & 1157 & 1890 & 1962 & 1741 & $2847(1.01)$ & 2904 & $2628(1.01)$ \\
\hline wing_nodal & 9290 & 9273 & 8465 & 13237 & 13258 & 12238 & 17899 & 17783 & 16258 (1.01) \\
\hline fe_4elt2 & 1152 & 1135 & 1039 & 1787 & 1796 & 1688 & $2765(1.01)$ & 2781 & 2590 \\
\hline vibrobox & 37441 & 43064 & 34392 & 46112 & 51006 & $47048(1.01)$ & $\mathbf{5 3 7 6 4}(1.01)$ & 58392 & 54503 \\
\hline bcsstk29 & 28151 & 28629 & 26055 & 41190 & 42935 & 38346 & $62891(1.01)$ & 63576 & 59548 \\
\hline 4 elt & 1056 & 1083 & 1005 & 1769 & 1766 & 1631 & 2953 & 2921 & $\mathbf{2 6 7 6}(1.01)$ \\
\hline fe_sphere & 2030 & 2037 & 1855 & 2913 & 2920 & 2701 & 4191 & 4151 & 3776 \\
\hline cti & 3181 & 3083 & 3033 & 4605 & 4532 & 4479 & 6461 & 6334 & 6181 \\
\hline memplus & 14942 & 16433 & 14097 & 17303 & 17936 & NAN & $19140(1.01)$ & 18978 & NAN \\
\hline $\operatorname{cs} 4$ & 2538 & 2552 & 2293 & 3579 & 3588 & 3137 & 4791 & 4817 & 4286 \\
\hline bcsstk30 & 77293 & 81069 & 79265 & 131405 & 128694 & 117414 & 191691 & 191445 & $175845(1.02)$ \\
\hline bcsstk31 & 27180 & 28557 & 25787 & 42645 & 45354 & 40029 & 66526 & 68375 & 61150 \\
\hline fe_pwt & 2933 & 3200 & 2857 & 6029 & 6036 & 6596 & 9310 & 9231 & 8487 \\
\hline bcsstk32 & 43371 & 47829 & 39902 & 70020 & 73377 & 64138 & 106733 & 108855 & 96197 \\
\hline fe_body & 2181 & 2947 & 2076 & 3424 & 4194 & 3290 & 5843 & 6326 & 5097 \\
\hline t60k & 998 & 977 & 937 & 1613 & 1594 & 1539 & 2484 & 2506 & 2345 \\
\hline wing & 4666 & 4671 & 4188 & 6700 & 6843 & 6067 & 9405 & 9308 & 8123 \\
\hline brack2 & 12655 & 13404 & 12240 & 19786 & 20172 & 18411 & 28872 & 29223 & 27130 \\
\hline finan512 & 1377 & 1296 & 1458 & 2592 & 2592 & 2592 & 10842 & 11962 & 11077 \\
\hline fe_tooth & 19346 & 20887 & 18336 & 29215 & 29849 & 26110 & 40162 & 40306 & 35988 \\
\hline fe_rotor & 23863 & 23936 & 21687 & 36225 & 36367 & 32746 & 53623 & 52497 & 48206 \\
\hline $598 \mathrm{a}$ & 28922 & 29674 & 26565 & 44760 & 45780 & 40980 & 64307 & 65094 & 57303 \\
\hline fe_ocean & 9613 & 9690 & 8397 & 14613 & 15059 & 13358 & 23317 & 22692 & 21212 \\
\hline Total & 2 & 1 & 27 & 3 & 2 & 26 & 3 & 3 & 24 \\
\hline
\end{tabular}

\subsection{Comparison with the best known partitions}

The second comparison is with the best balanced partitions reported at the Graph Partitioning archive. The majority of these best known results were generated with a very powerful algorithm presented by Soper et al. 25], which combines an evolutionary search approach with the JOSTLE multilevel procedure used as a black box. Since each run consists of 50,000 calls to JOSTLE, this approach requires significant running time of up to one week for large graphs. Another great portion of these best partitions were produced with the 


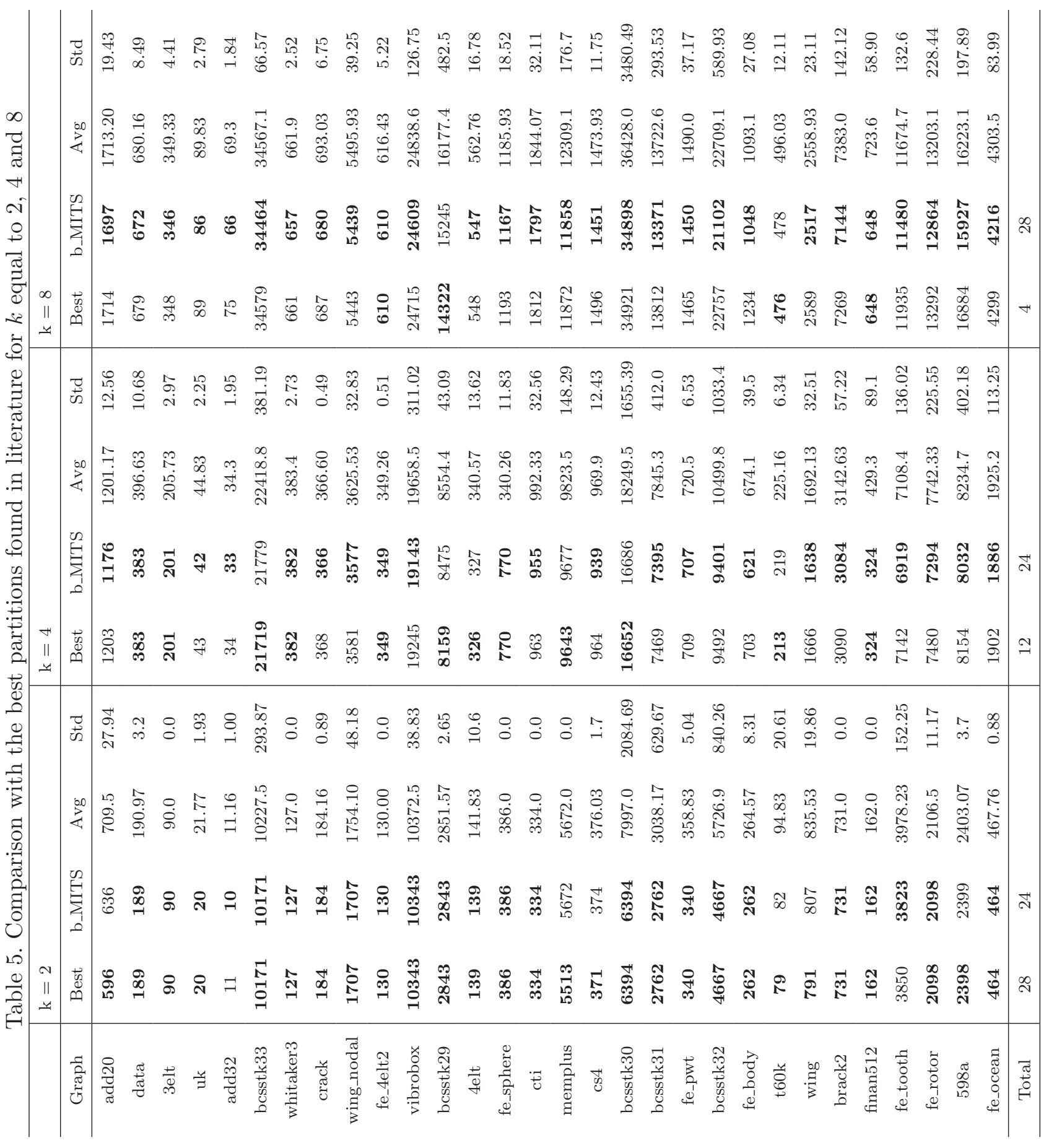




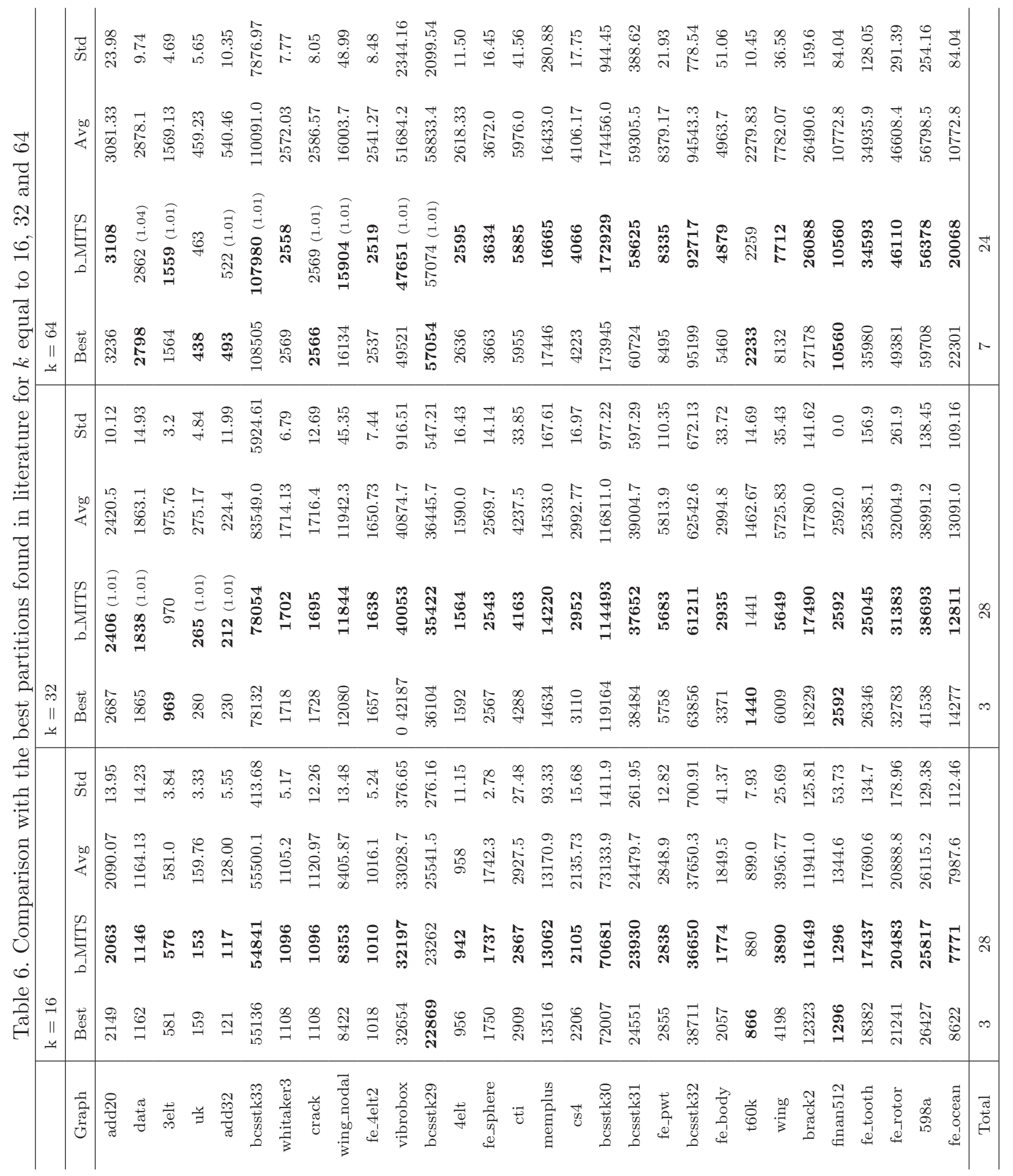


iterative multilevel algorithm by Walshaw [29] which also makes a number of calls to a multilevel procedure. The remaining best results are obtained with several other approaches [9,21,6]. It should be mentioned that the majority of these results were produced within very long computing time (up to one week for larger graphs).

Our second experiment assesses the performance of MITS algorithm in terms of partition quality relative to these state-of-the-art algorithms. For this experiment, we use cutoff time limits ranging from 1 minute for the smallest graph up to 1 hours for the largest one. Above this time limit, the algorithm does not produce significantly better results. Given its stochastic nature, the MITS algorithm is run 30 times for each graph and each value of $k$ (see Table 2).

Tables 5 and 6 give, for each graph and each value of $k$, the best results ever reported, the best results produced by our approach 2 , the average results as well as the standard deviations after 30 executions of the proposed algorithm. The row with heading Total gives the number of times our approach succeeded to reach or improve a best known up-to-date partition.

The results show that, in the case of bisection, our approach succeeded to reach the same solution quality of more than two thirds of the best reported balanced bisections. It also improved the best bisection in two cases. Interestingly, as $k$ increases ( $4 \leq k \leq 64$ ), our approach improved even $60 \%, 86 \%, 90 \%, 90 \%$ and $76 \%$ of the current best k-partitions where $k$ is equal to $4,8,16,32$ and 64 respectively.

As in the previous comparison, we note from Table 6 that in 12 cases where $k$ is equal to 32 and 64, our approach did not attain the perfect partition balance $(\varepsilon=1.00)$. However, this imbalance is generally low, with an average of 1.013 over these 12 imbalanced partitions. We also note that the number of imbalanced partitions is now slightly minimized compared to the first experiment when the running time was reduced. This implies that MITS sometimes requires more iterations to establish good balance. If the partitions are imbalanced, we compare them with the best known partitions of the same balance, which are also reported at the Graph Partitioning archive.

All these results show that the proposed approach is the overall winner when it comes to generating balanced k-partitions for these benchmark graphs.

$\overline{2}$ Results available at: http://www.info.univ-angers.fr/pub/hao/MITS.html 


\section{Analysis}

In this section, we turn our attention to the analysis of two important features of the MITS algorithm and try to answer the following questions: Is the perturbation mechanism (see Section 4.2) relevant for the MITS? Is the newly proposed Double move, which is used to define the neighborhood relation $N_{2}$ (see Section 4.1.2), a value-added one?

To answer these questions, we report in Table 7 the average results over 10 executions with MITS, when its two components (perturbation mechanism and neighborhood $N_{2}$ ) are included and excluded. We conduct these experiments with $k$ set to $4,8,16$ and 32 , on a set of 10 representative instances from the Graph Partitioning archive. For each $k$, colon " $+\left(P M \& N_{2}\right)$ " provides the average results when both the perturbation mechanism and the neighborhood $N_{2}$ are used within MITS. Colons " $-P M$ " and " $-N_{2}$ " report respectively the average results when the perturbation mechanism or the neighborhood $N_{2}$ is disabled. Finally, the last colon " $-\left(P M \& N_{2}\right)$ " gives the average results when both features are excluded from the MITS algorithm. The last row with heading Total shows the number of times each version of MITS produced the best average result over the 10 graphs.

From Table 7, we see that the performance of our MITS algorithm decreases when the perturbation mechanism or neighborhood $N_{2}$, or both are disabled. This confirms our previous observation that some diversification is needed to complement the aggressive tabu search strategy, which only considers border (critical) vertices. Indeed, although examining only border vertices for move consideration decreases the number of candidate moves at each iteration, this does not allow the search to escape from deep local optima. Therefore, this experiment confirms this intuitive explanation.

We can also see from Table 7 that in most cases, MITS produces significantly better partitions when the two neighborhoods $N_{1}$ and $N_{2}$ are jointly explored (see Section 4.1.2). This can be explained by the fact that neighborhood $N_{2}$ does not strictly impose the balance constraint as neighborhood $N_{1}$ does. With

$N_{2}$, there are more vertices which are considered to be moved to the target subset, introducing thus a greater degree of freedom during the search process. This complements the strict selection criterion of neighborhood $N_{1}$.

From Table 7 we can see that in several cases the simplest version of MITS, i.e. when both features are excluded, gives the best average results. However, as can be expected, the initial MITS still outperforms the simplest version in about $80 \%$ of cases. This implies that both of these elements are important for the efficiency of the proposed MITS algorithm. 
Table 7

The average results over 10 executions of MITS when the perturbation mechanism and the neighborhood relation $N_{2}$ are included and excluded.

\begin{tabular}{|c|c|c|c|c|c|c|c|c|}
\hline \multirow[b]{2}{*}{ Graph } & \multicolumn{4}{|l|}{$\mathrm{k}=4$} & \multicolumn{4}{|l|}{$\mathrm{k}=8$} \\
\hline & $+\left(P M \& N_{2}\right)$ & $-P M$ & $-N_{2}$ & $-\left(P M \& N_{2}\right)$ & $+\left(P M \& N_{2}\right)$ & $-P M$ & $-N_{2}$ & $-\left(P M \& N_{2}\right)$ \\
\hline 3elt & 206.1 & 253.0 & 223.2 & 255.3 & 349.8 & 359.3 & 372.2 & 377.1 \\
\hline uk & 44.9 & 64.0 & 47.7 & 61.9 & 92.2 & 95.5 & 98.3 & 102.5 \\
\hline bcsstk33 & 22180.8 & 22310.9 & 22166.9 & 22579.9 & 34581.5 & 34563 & 34598.3 & 34614.1 \\
\hline crack & 367.3 & 452.4 & 388.2 & 503.4 & 695.1 & 735.1 & 731.4 & 754.0 \\
\hline fe_4elt2 & 350.2 & 379.8 & 350.3 & 402.7 & 617.3 & 634.1 & 645.0 & 645.5 \\
\hline vibrobox & 19601.7 & 23114.2 & 21757.3 & 23649.7 & 24804.6 & 27726.1 & 27904.6 & 27405.0 \\
\hline 4 elt & 340.4 & 387.8 & 345.2 & 320.3 & 557.6 & 558.2 & 565.3 & 531.5 \\
\hline $\operatorname{cs} 4$ & 967.2 & 1093.6 & 980.6 & 1110.3 & 1478.6 & 1579.3 & 1485.2 & 1579.4 \\
\hline $\mathrm{t} 60 \mathrm{k}$ & 232.2 & 258 & 224.6 & 320.3 & 490.4 & 533.0 & 498.6 & 531.5 \\
\hline brack2 & 3149.4 & 4349.8 & 3150.3 & 4499.8 & 7404.2 & 7665.1 & 8489.7 & 7986.6 \\
\hline \multirow[t]{2}{*}{ Total } & 7 & 0 & 1 & 2 & 8 & 1 & 0 & 1 \\
\hline & $\mathrm{k}=16$ & & & & $\mathrm{k}=32$ & & & \\
\hline Graph & $+\left(P M \& N_{2}\right)$ & $-P M$ & $-N_{2}$ & $-\left(P M \& N_{2}\right)$ & $+\left(P M \& N_{2}\right)$ & $-P M$ & $-N_{2}$ & $-\left(P M \& N_{2}\right)$ \\
\hline 3elt & 582 & 586.4 & 630.8 & 625.6 & 976.9 & 992.3 & 984.8 & 980.6 \\
\hline uk & 160.8 & 171.0 & 163.7 & 171.8 & 275.9 & 284.2 & 292.0 & 288.2 \\
\hline bcsstk33 & 55572.8 & 62445 & 55541.3 & 55548.8 & 88996.9 & 85913.5 & 78891.5 & 79327.9 \\
\hline crack & 1116.7 & 1148.5 & 1184.9 & 1182.4 & 1713.4 & 1748.7 & 1820.1 & 1822 \\
\hline fe_4elt2 & 1015.4 & 1024.6 & 1020.4 & 1027.9 & 1652.0 & 1703.3 & 1680.2 & 1696 \\
\hline vibrobox & 33250.4 & 36704.9 & 33471.8 & 33621.2 & 41074.6 & 51313 & 40654.5 & 42962.5 \\
\hline $4 \mathrm{elt}$ & 962.5 & 960.6 & 962 & 932.5 & 1581.5 & 1638.6 & 1605.3 & 1506.4 \\
\hline $\operatorname{cs} 4$ & 2146.6 & 2248.4 & 2163 & 2253.6 & 3003.4 & 3294.5 & 3152.4 & 3159.2 \\
\hline $\mathrm{t} 60 \mathrm{k}$ & 901.8 & 895.0 & 908.3 & 932.5 & 1469.0 & 1484.0 & 1499.7 & 1506.4 \\
\hline brack2 & 11995.7 & 12536.1 & 11962.1 & 12197.0 & 17814.0 & 18691.5 & 17907.3 & 18051.5 \\
\hline Total & 6 & 1 & 1 & 2 & 6 & 0 & 2 & 1 \\
\hline
\end{tabular}

\section{Conclusion and remarks}

In this paper, we presented MITS, a multilevel iterated tabu search approach for balanced graph $k$-partitioning. The proposed algorithm follows the basic idea of the multilevel approach and integrates a new and powerful tabu search refinement procedure. The dedicated tabu search algorithm, which is the key component of the approach, includes a number of interesting features like the joint use of two neighborhoods, an adaptation of bucket sorting for incremental evaluation of move gains, guided selection strategies for vertex migration based on long term memory (move frequency) and a dynamic tabu tenure technique. To reinforce the diversification of the search process, the tabu search algorithm is combined with a perturbation mechanism to escape from deep local optima.

We assessed extensively the efficiency of the proposed algorithm with both short and long run times, on a collection of benchmark graphs provided by 
the Graph Partitioning Archive, with the cardinal number $k$ set to 2, 4, 8, 16 , 32 and 64 . The results generated in short computation time (from 1 second up to 2.5 minutes) are far better than those produced by the two well-known partitioning packages METIS or CHACO. When the running time is prolonged from 1 minute up to 1 hour, our approach succeeds even to improve more than two thirds of the best balanced partitions ever reported in the literature (which were often obtained with much longer computing time up to one week).

Finally, it is recognized that there may be a significant trade-off between partition quality and balance. Indeed, allowing a certain degree of imbalance usually leads to partitions of better quality. In this work, we aimed to produce perfectly balanced partitions, and did not specifically address the possibility to allow imbalance up to a certain limit. Yet, we tested our MITS algorithm with the balance constraint relaxed up to certain degree. We observed that the quality of these imbalanced partitions, even if they are not reported here, remains highly competitive with respect to the best known partitions of the same imbalance. In fact, we managed to improve once again a number of the current best partitions, although the percentage of these improved partitions was lower than in the case of balanced partitions.

\section{Acknowledgment}

We are grateful to the referees for their comments and questions which helped us to improve the paper. The work is partially supported by the Pays de la

Loire Region (France) within the projects RaDaPop projects (2009-2013) and LigeRO (2010-2013).

\section{References}

[1] C.J. Alpert and A.B. Kahng. Recent directions in netlist partitioning. Integration: the VLSI Journal, 19(12): 1-81, 1995.

[2] S.T. Barnard and H.D. Simon. A fast multilevel implementation of recursive spectral bisection for partitioning unstructured problems. Concurrency: Practice 83 Experience, 6(2): 101-117, 1994.

[3] R. Battiti and A. Bertossi. Greedy and prohibition-based heuristics for graph partitioning. IEEE Transactions on Computers, 48: 361-385, 1999.

[4] T.N. Bui and C. Jones A heuristic for reducing fill-in in sparse matrix factorization. In R.F Sincovec et al. (Eds), Parallel Processing for Scientific Computing, SIAM, Philadelphia, 445-452, 1993. 
[5] T.N. Bui and B.R. Moon. Genetic algorithm and graph partitioning. IEEE Transactions on Computers, 45: 841-855, 1996.

[6] P. Chardaire, M. Barake, and G. P. McKeown. A PROBE based heuristic for Graph Partitioning. IEEE Transactions on Computers, 56(12): 1707-1720, 2007.

[7] M. Dell'Amico and F. Maffioli. A Tabu Search approach to the 0-1 equicut problem. In Meta Heuristics 1995: The State of the Art, Kluwer Academic Publishers, 361-377, 1996.

[8] B. Hendrickson and R. Leland. A multilevel algorithm for partitioning graphs. In S. Karin. (Eds) Proceedings of the 1995 ACM/IEEE Conference on Supercomputing, San Diego, ACM Press, New York, 1995.

[9] M. Holtgrewe, P. Sanders, and C. Schulz. Engineering a Scalable High Quality Graph Partitioner. Technical Report, 2009.

[10] C. Fiduccia and R. Mattheyses. A linear-time heuristics for improving network partitions. In: Proceedings of the 19th Design Automation Conference, 171-185, 1982.

[11] M. Garey and D. Johnson. Computers \& Intractability: A Guide to the Theory of NP-Completeness. W.H. Freeman and Company, 1979.

[12] L. Di Gaspero and A. Schaerf. Neighborhood portfolio approach for local search applied to timetabling problems. Journal of Mathematical Modeling and Algorithms, 5(1): 65-89, 2006.

[13] F. Glover and M. Laguna. Tabu Search. Kluwer Academic Publishers, Boston, 1997.

[14] B. Hendrickson and T.G. Kolda. Graph partitioning models for parallel computing. Parallel Computing, 26(12): 1519-1534, 2000.

[15] D.S. Johnson, C.R. Aragon, L.A. Mcgeoch and C. Schevon. Optimization by simulated annealing: an experimental evaluation; Part-I, graph partitioning. Operations Research, 37: 865-892, 1989.

[16] G. Karypis and V. Kumar. MeTiS 4.0: Unstructured graphs partitioning and sparse matrix ordering system. Technical Report, Department of Computer Science, University of Minnesota, 1998.

[17] G. Karypis and V. Kumar. A fast and high quality multilevel scheme for partitioning irregular graphs. SIAM Journal on Scientific Computing, 20(1): 359-392, 1998.

[18] G. Karypis and V. Kumar. Multilevel k-way partitioning scheme for irregular graphs. Journal of Parallel and Distributed Computing, 48(1): 96-129, 1998.

[19] B.W. Kernighan and S. Lin. An efficient heuristic procedure for partitioning graphs. Bell System Technical Journal, 49, 291-307, 1970. 
[20] Peter Merz and Bernd Freisleben. Fitness Landscapes, Memetic Algorithms and Greedy Operators for Graph Bi-Partitioning. Evolutionary Computation, 8(1): 6191, 2000.

[21] H. Meyerhenke, B. Monien, and T. Sauerwald. A new diffusion-based multilevel algorithm for computing graph partitions of very high quality. In Proceedings of the 22nd International Parallel and Distributed Processing Symposium (IPDPS'08), 2008.

[22] C. Papadimitriou and K. Steiglitz. Combinatorial Optimization: Algorithms and Complexity. Prentice-Hall, 1982.

[23] E. Rolland, H. Pirkul and F. Glover. Tabu search for graph partitioning. Annals of Operations Research, 63, 209-232, 1996.

[24] J. Shi and J. Malik. Normalized cuts and image segmentation. Proceedings of the IEEE Conference on Computer Vision and Pattern Recognition, pages 731-737, 1997.

[25] A.J. Soper, C. Walshaw, and M. Cross. A combined evolutionary search and multilevel optimisation approach to graph-partitioning. Journal of Global Optimization, 29(2): 225-241, 2004.

[26] A. Slowik and M. Bialko. Partitioning of VLSI circuits on subcircuits with minimal number of connections using evolutionary algorithm. In L. Rutkowski et al. (Eds.), ICAISC 2006, Lecture Notes in Artificial Intelligence 4029, pages 470-478, Springer-Verlag, 2006.

[27] E.-G. Talbi and P. Bessiere. A parallel genetic algorithm for the graph partitioning problem. Proceedings of the 5th International Conference on Supercomputing, pages 312 - 320, 1991.

[28] C. Walshaw and M. Cross. Mesh partitioning: a multilevel balancing and refinement algorithm. SIAM Journal on Scientific Computing, 22: 63-80, 2000.

[29] C. Walshaw. Multilevel refinement for combinatorial optimisation problems. Annals of Operations Research, 131: 325-372, 2004.

[30] H. Zha, X. He, C. Ding, H. Simon, and M. Gu. Bipartite graph partitioning and data clustering. Proceedings of the ACM 10th International Conference on Information and Knowledge (CIKM 2001), pages 25-31, 2001. 